\title{
Early pharmacologic conversion of atrial fibrillation after off-pump coronary artery bypass grafting
}

\author{
Heemoon Lee ${ }^{1 \#}$, Hee Jung Kim ${ }^{2 \#}$, Jae Suk Yoo ${ }^{1}$, Dong Jin Kim ${ }^{1}$, Sang Youn Yeom ${ }^{1}$, Kwang Ree Cho ${ }^{1}$ \\ ${ }^{1}$ Department of Thoracic and Cardiovascular Surgery, Sejong General Hospital, Bucheon, Korea; ${ }^{2}$ Department of Thoracic and Cardiovascular \\ Surgery, Korea University Anam Hospital, Seoul, Korea \\ Contributions: (I) Conception and design: KR Cho; (II) Administrative support: KR Cho, JS Yoo, DJ Kim; (III) Provision of study materials or \\ patients: KR Cho, SY Yeom, HJ Kim; (IV) Collection and assembly of data: KR Cho, H Lee, SY Yeom; (V) Data analysis and interpretation: KR \\ Cho, H Lee, HJ Kim; (VI) Manuscript writing: All authors; (VII) Final approval of manuscript: All authors. \\ \#These authors contributed equally to this work. \\ Correspondence to: Kwang Ree Cho, MD, PhD. Department of Thoracic and Cardiovascular Surgery, Sejong General Hospital, 28 , Hohyeon-ro 489 \\ beon-gil, Bucheon-si, Gyeonggi-do 14754, Republic of Korea. Email: ckrym@hanmail.net.
}

\begin{abstract}
Background: The aim of this study was to evaluate the efficacy of early amiodarone-based pharmacological cardioversion for postoperative atrial fibrillation (POAF) following off-pump coronary bypass grafting (OPCAB).

Methods: A total of 507 patients who underwent OPCAB between 2015 and 2017 were categorized into POAF ( $\mathrm{n}=94)$ and no-POAF ( $\mathrm{n}=413)$ groups. Patients in the POAF group were treated according to the following institutional protocol: $150 \mathrm{mg}$ loading dose of intravenous amiodarone, followed by oral administration with sequential maintenance doses at 600, 400, and $200 \mathrm{mg}$ per day. If sinus rhythm was restored before discharge, patients were discharged without amiodarone or anticoagulants, except for dual antiplatelets.

Results: Before discharge at index hospitalization, 97.8\% of POAF patients had restored sinus rhythm. Independent risk factors for POAF were age, unstable angina, prior percutaneous transluminal coronary angioplasty, and left atrial diameter. The mean follow-up duration was $41.1 \pm 12.8$ months. Freedom from overall mortality and composite events, including mortality, major bleeding requiring admission and cerebrovascular events, were similar between the 2 groups. Results were consistent after propensity-score matching.

Conclusions: Amiodarone-based rapid pharmacological cardioversion of POAF resulted in a high sinus rhythm conversion rate (97.9\%). Rate of late adverse cardiovascular events including stroke, were low even without anticoagulation. As optimal treatment and anticoagulation guidelines for POAF after OPCAB have not yet been established, amiodarone-based treatment protocols may be considered as a useful option.
\end{abstract}

Keywords: Arrhythmia; arrhythmia therapy; atrial fibrillation; coronary artery bypass grafting

Submitted Mar 07, 2021. Accepted for publication May 27, 2021.

doi: $10.21037 /$ jtd-21-466

View this article at: https://dx.doi.org/10.21037/jtd-21-466

\section{Introduction}

Postoperative atrial fibrillation (POAF) is a frequent complication following cardiac surgery, with an incidence of up to $40 \%$. Most studies have ascertained that POAF is associated with adverse clinical outcomes in both the early and late periods (1-5). However, studies regarding the effects of POAF on long-term clinical outcomes have inherent confounding biases and are at risk of misunderstanding. The predictors of POAF were similar to those of long-term mortality (6). POAF may not be the cause of mortality, but likely the result of preoperative risk factors. In addition, POAF may be associated with 
various perioperative conditions including transfusion due to bleeding, preconditioned heart fibrosis, inflammatory reaction associated with cardiopulmonary bypass, and poor intraoperative conditions such as myocardial damage. Thus, POAF development may be due to complications of the operation itself rather than underlying conditions $(7,8)$. However, the previously reported risk factors have not been consistent. Furthermore, POAF following offpump coronary bypass grafting (OPCAB) may be due to an increased susceptibility to triggers of atrial fibrillation (AF) after operation, rather than a structural substrate before operation or surgical trauma (9).

Other criticisms concern the benign and self-limiting nature of POAF $(6,10)$. Considering its high conversion rate and short duration of presentation, the direct impact of POAF on late adverse events is uncertain. Therefore, determining the association between POAF and adverse clinical outcomes can be challenging.

In addition, consensus guidelines on the optimal treatment and anticoagulation strategies for POAF have not yet been established (11-13). Patients indicated for coronary artery bypass grafting (CABG) usually have multiple comorbidities and high risk for cardiovascular events requiring several medications including antiplatelet agents. Additional anticoagulants should therefore be administered cautiously.

In our institution, we proposed an amiodarone-based institutional treatment protocol for POAF after OPCAB. Patients with POAF were treated with amiodarone, and were discharged without anticoagulation when sinus rhythm was achieved.

The aim of our study was to evaluate the efficacy of early amiodarone-based pharmacological cardioversion of POAF following OPCAB.

We present the following article in accordance with the STROBE reporting checklist (available at https://dx.doi. org/10.21037/jtd-21-466).

\section{Methods}

\section{Study population}

We retrospectively reviewed data from January 2015 through December 2017 of 507 patients who underwent $\mathrm{OPCAB}$ in our institution; those with a history of persistent POAF or paroxysmal AF were excluded. Included subjects were then classified into either the POAF ( $\mathrm{n}=94)$ or the noPOAF $(n=413)$ group. The mean age was $63.8 \pm 9$ years, and the mean $\mathrm{CHA}_{2} \mathrm{DS}_{2}-\mathrm{VAS}_{\mathrm{C}}$ score was $2.5 \pm 1.5$. The study was conducted in accordance with the Declaration of Helsinki (as revised in 2013). The study protocol was approved by the hospital's institutional review board, which waived the requirement for patient consent (IRB no: 2020-0643, approval date: 11-18-2020).

\section{Operative procedures and routine postoperative managements}

The main strategy of CABG in our institution is OPCAB via standard median sternotomy with skeletonized bilateral internal thoracic artery. Composite Y or I grafts were constructed after harvesting. The in situ left internal thoracic artery was anastomosed to the left anterior descending artery in most cases; otherwise, the right internal thoracic artery was used. The remaining left circumflex artery or territories were revascularized using a sequential anastomosis technique. Bilateral internal thoracic artery and saphenous vein was used in 1 patient. Single internal thoracic artery and saphenous vein was used in 2 patients.

Routine medication included oral aspirin $(100 \mathrm{mg})$, clopidogrel $(75 \mathrm{mg})$, and statins. Aspirin was administered on the day of operation, and readministered within 6 hours after operation. Clopidogrel and statins were started at postoperative day 1. Prophylactic beta-blockers, calcium channel blockers and amiodarone for preventing POAF were not included as routine medication. Patients were continuously monitored via cardiac telemetry either in the intensive care unit or in the general ward. Twelve-lead electrocardiography (ECG) was performed on patients in whom POAF was detected on telemetry. Those with documented AF were then treated according to the institutional protocol described below. Daily ECGs were taken in patients with POAF. ECGs were also regularly taken at 1, 3 months post-discharge and then every 3 to 6 months.

\section{Protocol for POAF}

When POAF was documented, patients were treated with an amiodarone-based institutional protocol as in the following description. (I) An initial loading dose of $150 \mathrm{mg}$ intravenous amiodarone. If patients had heart rate $>120 / \mathrm{min}$ with palpitations, an additional bolus of $150 \mathrm{mg}$ amiodarone was considered. (II) Sequential maintenance doses at $600,400,200 \mathrm{mg}$ oral amiodarone per day. If heart rate $<60 /$ min, maintenance therapy was omitted. (III) In patients with prolonged POAF of $>6$ hours, anticoagulation with 
low molecular weight heparin or unfractionated heparin was initiated (target activated thromboplastin time: 40-50 sec in patients with chronic kidney disease). Oral aspirin and new oral anticoagulants (NOAC) were considered when AF persisted until the day of discharge. (IV) Amiodarone and oral anticoagulation agents were discontinued unless $\mathrm{AF}$ was present at discharge.

Electrical cardioversion was considered if patients were symptomatic with systolic blood pressure $<80 \mathrm{mmHg}$.

\section{Follow-up}

Hospital records were reviewed retrospectively. The primary endpoint was recurrence of AF during followup. The secondary endpoints were all-cause mortality and cerebrovascular events (CVA). Early mortality was defined as death during hospitalization. Composite endpoints included mortality, major bleeding requiring admission, and CVA.

Follow-up survival data were available for all patients (85.8\% from our hospital, $14.2 \%$ from the national registry). The mean follow-up duration for all patients was 41.1 \pm 12.8 [median (Q1-Q3), 41.5 (32.7-51.1)] months; while that for the POAF and the no-POAF group was $38.5 \pm 13.8$ [39.5 (29.0-49.1)] months and 41.7 \pm 12.5 [42.8 (33.5-51.3)] months, respectively $(\mathrm{P}=0.028)$.

\section{Statistical analysis}

Categorical variables are presented as frequencies and percentages, while continuous variables are presented as means with standard deviations, or medians and Q1Q3 quartiles. Inter-group differences were assessed using the $t$-test (or the Mann-Whitney test when the normality assumption was in doubt) and the Chi-square test (or the Fisher's exact test when the expected cell frequency was $<5$ ). To balance the distribution of baseline risk factors between the groups, inverse probability of treatment weighting (IPTW)-adjusted analysis was performed. Propensity score (PS) was obtained by multiple logistic regression, which was based on preoperative baseline characteristics including age, sex, body surface area, body mass index, hypertension, diabetes mellitus, stroke, New York Heart Association functional class, Canadian Cardiovascular Society class 4 angina, preoperative beta-blocker use, hyperthyroidism, chronic renal failure, chronic obstructive pulmonary disease, $\mathrm{CHA}_{2} \mathrm{DS}_{2}-\mathrm{VAS}_{\mathrm{C}}$ score, peripheral occlusive arterial disease, cancer history, old myocardial infarction, percutaneous transluminal coronary angioplasty (PTCA), left ventricular ejection fraction of $<40 \%$, left atrial (LA) enlargement (LA diameter $\geq 40 \mathrm{~mm}$ ), preoperative intra-aortic ballooning pump support, unstable angina, stable angina, non-ST elevation myocardial infarction, triple vessel disease, left main disease, and the number of anastomoses. Weights for POAF patients were the inverse of the PS, and those for patients without POAF were the inverse of 1-PS. To reduce variability in the IPTW models, stabilized weights were used (14). We also analyzed the PS matching as an added robust analysis result. For PS matching, ninety-one patients with POAF were matched 1:1 with patients without POAF using the nearest-neighbor matching without replacement method, with a matching tolerance (caliper) of 0.25 . Survival curves were generated using the Kaplan-Meier method, and survival rates were compared between the 2 groups using the log-rank test. The Cox proportional hazards model analysis was employed to estimate the treatment effect of POAF versus no-POAF on long-term clinical outcomes in terms of overall mortality and composite endpoints. Hazard ratios (HRs) of late clinical outcomes between the 2 groups were compared based on original unmatched data, IPTW models, and matched data. $\mathrm{P}$ values $<0.05$ were considered statistically significant. Rates of missingness for data in our models were $<1 \%$, and no imputation was performed for missing data. All statistical analyses were performed using the R3.6.3 software (R Foundation for Statistical Computing, Vienna, Austria).

\section{Results}

\section{Baseline characteristics and operative data}

Table 1 summarizes the baseline characteristics of the study population before and after IPTW adjustments. Patients with POAF tended to have older age, lower rates of dyslipidemia, higher PTCA history, larger LA diameter, higher prevalence of diabetes mellitus, and higher history of CVA as compared with no-POAF patients. After IPTW adjustment, no differences in the demographic data were observed. Based on operative data, POAF patients showed higher incidences of unstable and stable angina. However, no differences were observed after IPTW adjustment (Table 2). It was consistent in matched data, described in the supplementary appendix (Tables S1,S2).

\section{Protocol-based treatment for POAF}

The time to develop POAF after surgery was $1.9 \pm 1.0$ 
Table 1 Baseline characteristics

\begin{tabular}{|c|c|c|c|c|c|c|c|c|}
\hline Variables & \multicolumn{4}{|c|}{ Before IPTW } & \multicolumn{4}{|c|}{ After IPTW* } \\
\hline Age, years & $\begin{array}{c}63.0 \\
(56.0-70.0)^{b}\end{array}$ & $\begin{array}{c}68.1 \\
(62.0-74.2)^{b}\end{array}$ & & & $\begin{array}{c}63.8 \\
(56.4-71.0)^{b}\end{array}$ & $\begin{array}{c}62.6 \\
(54.9-68.5)^{\mathrm{b}}\end{array}$ & & \\
\hline \multirow[t]{2}{*}{ BSA, $m^{2}$} & $1.72 \pm 0.17^{\mathrm{a}}$ & $1.71 \pm 0.18^{a}$ & 0.494 & 0.077 & $1.72 \pm 0.17^{\mathrm{a}}$ & $1.75 \pm 0.18^{\mathrm{a}}$ & 0.254 & 0.182 \\
\hline & $\begin{array}{c}1.73 \\
(1.61-1.83)^{b}\end{array}$ & $\begin{array}{c}1.73 \\
(1.58-1.85)^{b}\end{array}$ & & & $\begin{array}{c}1.72 \\
(1.61-1.83)^{b}\end{array}$ & $\begin{array}{c}1.75 \\
(1.62-1.87)^{\mathrm{b}}\end{array}$ & & \\
\hline $\mathrm{BMI}, \mathrm{kg} / \mathrm{m}^{2}$ & $24.8 \pm 3.1^{\mathrm{a}}$ & $24.7 \pm 2.7^{\mathrm{a}}$ & 0.725 & 0.199 & $24.8 \pm 3.0^{\mathrm{a}}$ & $25.1 \pm 2.7^{\mathrm{a}}$ & 0.539 & 0.095 \\
\hline Diabetes mellitus, $\mathrm{n}(\%)$ & $208(50.4)$ & $58(61.7)$ & 0.061 & 0.230 & $217(52.5)$ & $47(50.5)$ & 0.786 & 0.044 \\
\hline Stroke, n (\%) & $234(56.7)$ & $61(68.1)$ & 0.055 & 0.238 & $241(58.4)$ & $49(52.7)$ & 0.509 & 0.108 \\
\hline Dyslipidemia, n (\%) & $132(32.0)$ & $16(17.0)$ & 0.006 & 0.353 & $121(29.3)$ & $27(29.0)$ & 0.892 & 0.024 \\
\hline NYHA class III-IV, n (\%) & $18(4.4)$ & $5(5.3)$ & 0.897 & 0.045 & $18(4.4)$ & $6(6.5)$ & 0.595 & 0.079 \\
\hline CCS class 4, n (\%) & $20(4.8)$ & $7(7.4)$ & 0.447 & 0.109 & $22(5.3)$ & $4(4.3)$ & 0.745 & 0.036 \\
\hline Preoperative beta-blocker use, n (\%) & $310(75.1)$ & $70(74.5)$ & $>0.999$ & 0.014 & $311(75.3)$ & $71(76.3)$ & 0.764 & 0.042 \\
\hline Hyperthyroidism, n (\%) & $6(1.5)$ & $0(0.0)$ & 0.518 & 0.172 & $5(1.2)$ & $0(0.0)$ & 0.258 & 0.155 \\
\hline PAOD, n (\%) & $134(32.4)$ & $34(36.2)$ & 0.568 & 0.079 & $135(32.7)$ & $27(29.0)$ & 0.608 & 0.075 \\
\hline Cancer, n (\%) & $16(3.9)$ & $8(8.5)$ & 0.101 & 0.193 & $18(4.3)$ & $3(3.2)$ & 0.694 & 0.038 \\
\hline OMI history, n (\%) & $26(6.3)$ & $11(11.7)$ & 0.110 & 0.190 & $32(7.7)$ & $8(8.6)$ & 0.824 & 0.029 \\
\hline PTCA history, n (\%) & $72(17.4)$ & $30(31.9)$ & 0.003 & 0.341 & $85(20.6)$ & $18(19.4)$ & 0.775 & 0.035 \\
\hline$E F<40, n(\%)$ & $84(20.3)$ & $16(17.0)$ & 0.558 & 0.085 & $82(19.9)$ & $23(24.7)$ & 0.520 & 0.120 \\
\hline LA enlargement, $\mathrm{n}(\%)$ & $200(48.4)$ & $64(68.1)$ & 0.001 & 0.407 & $216(52.3)$ & $51(54.8)$ & 0.710 & 0.061 \\
\hline
\end{tabular}

*, counts in the weighted cohort may not sum up to the expected totals owing to rounding. Percentages may not total to 100 because of rounding. Disagreements between numbers and percentages in the weighted cohort are therefore the result of rounding of non-integer number values. ${ }^{a}$ Mean \pm standard deviation; ${ }^{b}$ Median (Q1-Q3). IPTW, inverse probability of treatment weighting; POAF, postoperative atrial fibrillation; SMD, standardized mean difference; BSA, body surface area; BMI, body mass index; NYHA, New York Heart Association; CCS, Canadian Cardiovascular Society; COPD, chronic obstructive pulmonary disease; POAD, peripheral occlusive arterial disease; OMI, old myocardial infarction; PTCA, percutaneous transluminal coronary angioplasty; EF, ejection fraction; LA, left atrial. 
Table 2 Operative data

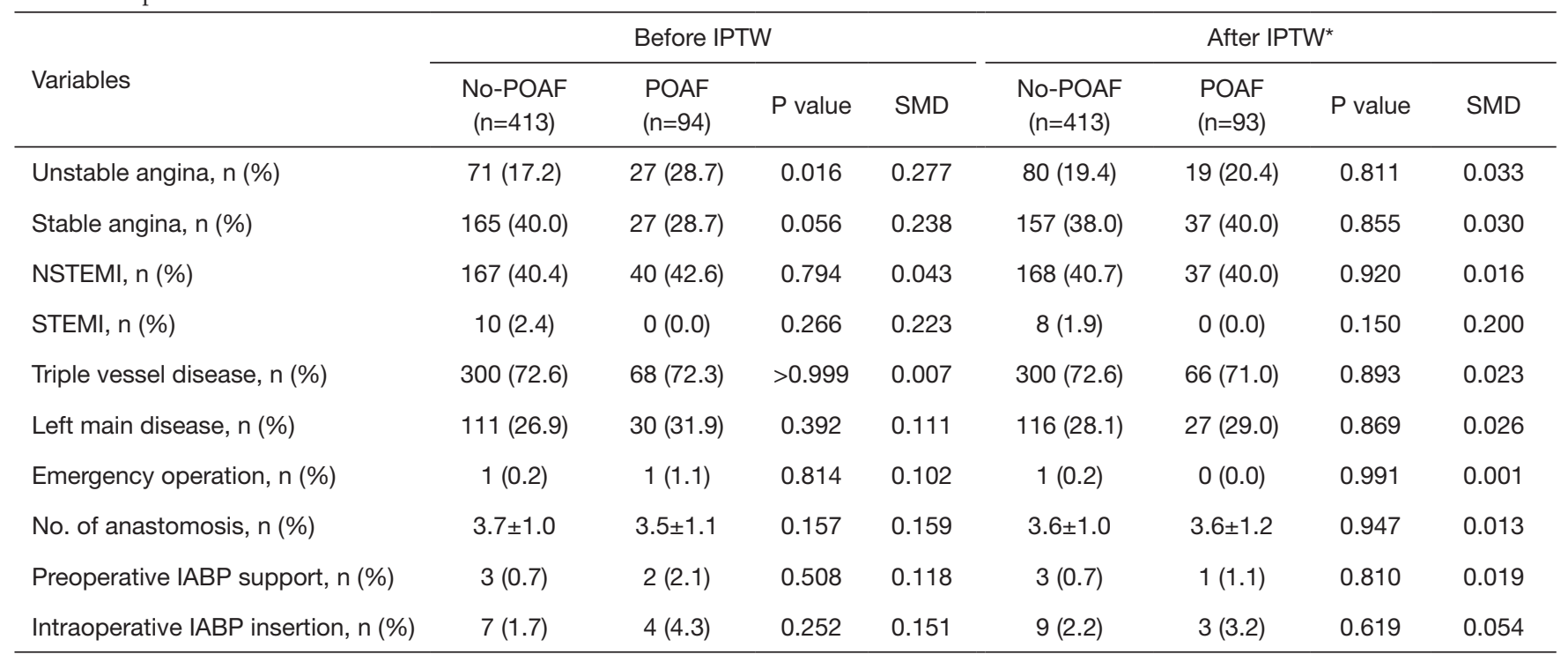

*, counts in the weighted cohort may not sum up to the expected totals owing to rounding. Percentages may not total to 100 because of rounding. Disagreements between numbers and percentages in the weighted cohort are therefore the result of rounding of non-integer number value. IPTW, inverse probability of treatment weighting; POAF, postoperative atrial fibrillation; SMD, standardized mean difference; NSTEMI, non-ST-elevation myocardial infarction; STEMI, ST-elevation myocardial infarction; IABP, intra-aortic balloon pump.

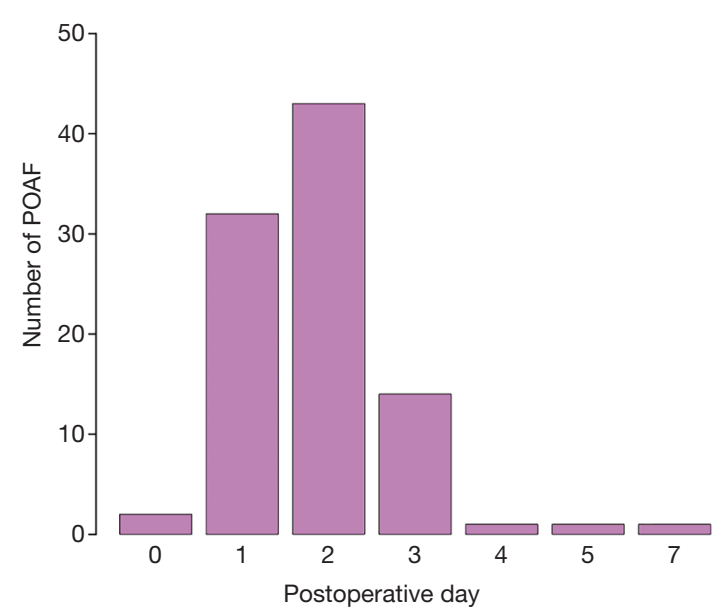

Figure 1 Incidence of postoperative atrial fibrillation according to postoperative days. POAF, postoperative atrial fibrillation.

$\{2[1-2]\}$ days, with postoperative day 2 being the most common time for POAF development (Figure 1). A total of $94.7 \%$ of POAF patients ( $n=89)$ were treated according to the amiodarone-based institutional protocol. Among them, sinus rhythm was achieved in $97.9 \%$, and the time to sinus rhythm conversion was $1.6 \pm 2.4\{1[0-2.75]\}$ days. Only 2 POAF patients $(2.1 \%)$ were discharged without sinus rhythm conversion, and were administered NOAC, respectively, upon discharge. Among them, AF was persisted in one patient, sinus rhythm conversion was occurred in the other patient. During follow-up, AF recurrence was occurred in two patients. Consequently, persistent AF was noted in 3 patients.

\section{Early outcomes}

Before IPTW adjustment, 8 early deaths (1.9\%) in the no-POAF group and $1(1.1 \%)$ in the POAF group were reported $(\mathrm{P}>0.999)$. Early postoperative mortality and morbidity rates were not significantly different between the 2 groups both before and after IPTW adjustment (Table 3).

\section{Late outcomes}

Three-year freedom from overall mortality did not differ between the no-POAF and POAF groups (93.2\% vs. 89.0\%, respectively, $\mathrm{P}=0.374)$. Three-year freedom from composite events also did not differ between groups (88.8\% vs. 83.7\%, respectively, $\mathrm{P}=0.356$ ) (Figure 2).

The IPTW-weighted Kaplan-Meier analysis also showed no difference in freedom from overall mortality and composite events $(\mathrm{P}=0.397$ and $\mathrm{P}=0.716$, respectively, Figure 3).

Figure 4 summarizes the HRs for clinical outcomes 
Table 3 Early outcomes

\begin{tabular}{|c|c|c|c|c|c|c|c|c|}
\hline Variables & \multicolumn{4}{|c|}{ Before IPTW } & \multicolumn{4}{|c|}{ After IPTW* } \\
\hline Early mortality, n (\%) & $7(1.7)$ & $1(1.1)$ & $>0.999$ & 0.054 & $8(1.9)$ & $1(1.1)$ & 0.272 & 0.112 \\
\hline Stroke, n (\%) & $11(2.7)$ & $3(3.2)$ & $>0.999$ & 0.031 & $11(2.7)$ & $3(3.2)$ & 0.813 & 0.028 \\
\hline PMI, n (\%) & $3(0.7)$ & $0(0.0)$ & 0.933 & 0.121 & $3(0.7)$ & $0(0.0)$ & 0.418 & 0.123 \\
\hline Bleeding reoperation, n (\%) & $3(0.7)$ & $2(2.1)$ & 0.508 & 0.118 & $4(0.9)$ & $1(1.1)$ & 0.666 & 0.042 \\
\hline Mediastinitis, n (\%) & $7(1.7)$ & $3(3.2)$ & 0.596 & 0.097 & $7(1.7)$ & $4(4.3)$ & 0.256 & 0.134 \\
\hline
\end{tabular}

*, counts in the weighted cohort may not sum up to the expected totals owing to rounding. Percentages may not total to 100 because of rounding. Disagreements between numbers and percentages in the weighted cohort are therefore the result of rounding of non-integer number values. IPTW, inverse probability of treatment weighting; POAF, postoperative atrial fibrillation; SMD, standardized mean difference; PMI, perioperative myocardial infarction; LCOS, low cardiac output syndrome.
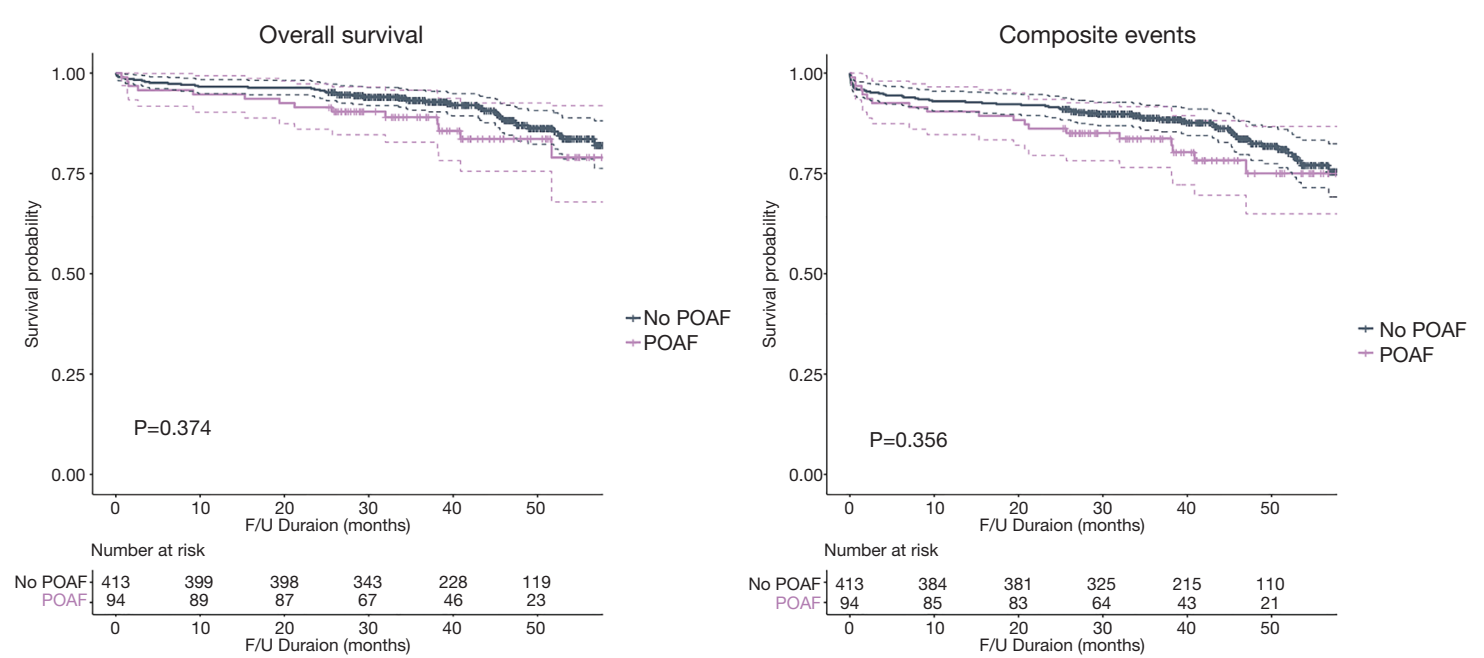

Figure 2 Kaplan-Meier curves of POAF and no-POAF patients in the total population. Kaplan-Meier curves for comparison of overall survival and composite events including mortality, bleeding, and cerebrovascular event between POAF and no-POAF patients after offpump coronary bypass grafting in the total population. POAF, postoperative atrial fibrillation.

between the 2 groups. Various statistical methods consistently indicated no differences in overall mortality and composite events between the 2 groups.

\section{Risk factors for developing $P O A F$}

Table 4 presents the results of the univariable and multivariable logistic regression analyses for the assessment of risk factors for POAF. Multivariable analysis revealed that age [odds ratio, $\mathrm{OR}=1.054,95 \%$ confidence interval (CI): $1.027-1.083, \mathrm{P}<0.001]$, unstable angina $(\mathrm{OR}=1.805,95 \%$ CI: 1.036-3.098, $\mathrm{P}=0.034)$, PTCA history $(\mathrm{OR}=2.003,95 \%$
CI: 1.172-3.384, $\mathrm{P}=0.010)$, and LA enlargement $(\mathrm{OR}=2.141$, 95\% CI: $1.312-3.560, \mathrm{P}=0.003)$ were significant risk factors for POAF. Dyslipidemia showed a preventive effect against POAF (OR=0.472, 95\% CI: 0.250-0.844, $\mathrm{P}=0.014)$.

\section{Discussion}

We found that the incidence of POAF following isolated OPCAB was $18.5 \%$ of the entire cohort. Sinus rhythm conversion rate in POAF patients treated according to the amiodarone-based institutional protocol was $97.9 \%$. Only 3 persistent $\mathrm{AF}$ cases were observed during follow-up. Even 

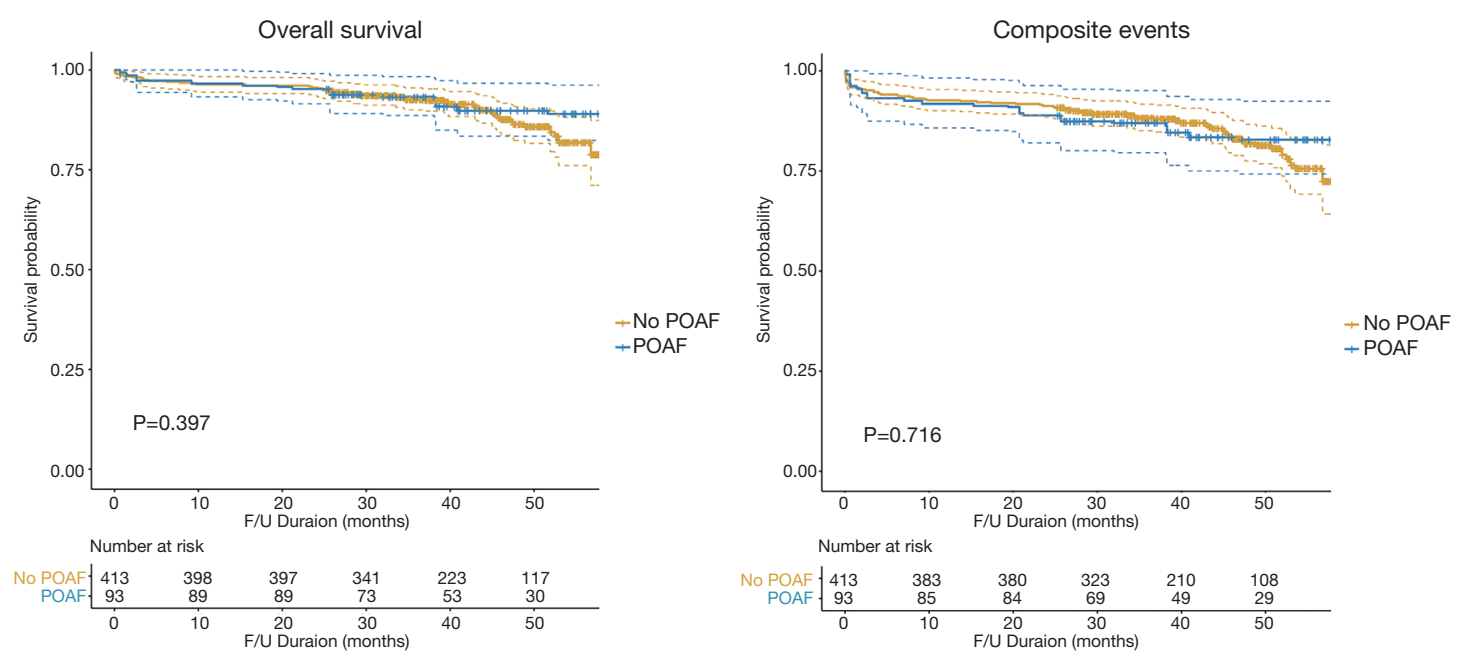

Figure 3 Kaplan-Meier curves of POAF and no-POAF patients in the IPTW adjusted population. Kaplan-Meier curves for comparison of overall survival and composite events including mortality, bleeding, and cerebrovascular event between POAF and no-POAF patients after off-pump coronary bypass grafting in the IPTW adjusted population. POAF, postoperative atrial fibrillation; IPTW, inverse probability of treatment weighting.

Overall survival

Methods
Crude
Multivariable Cox regression
IPTW adjusted analysis
PS matched analysis

Composite events

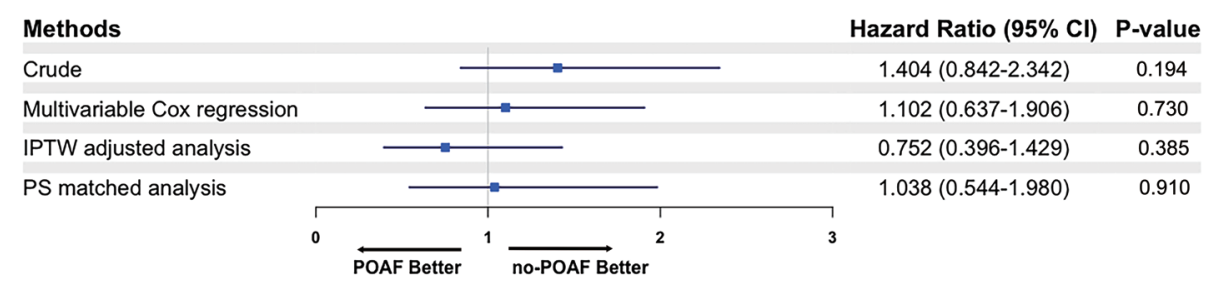

Figure 4 Forest plot of hazard ratios for late clinical outcomes by various methods. Forest plot of hazard ratios for overall survival and composite events including mortality, bleeding, and cerebrovascular event between POAF and no-POAF patients after off-pump coronary bypass grafting by various methods; Univariable Cox regression (Crude), Multivariate Cox regression, Inverse probability of treatment weighting adjusted analysis, Propensity score matched analysis. POAF, postoperative atrial fibrillation.

without anticoagulation or amiodarone medication after discharge, the risk of mid-term outcomes (overall mortality and composite event) of the POAF and no-POAF groups did not differ significantly.

Adverse effects of POAF following CABG have been reported. A recent meta-analysis showed that POAF associated with reduced 10 -year survival rates, and significantly higher 30-day mortality, stroke, respiratory failure, pneumonia and hospitalization (1). Villareal et al. showed results of 6,475 patients who underwent CABG 
Table 4 Multivariable logistic regression for risk factor analysis of POAF

\begin{tabular}{lccc}
\hline Variables & OR & $95 \% \mathrm{Cl}$ & $\mathrm{P}$ value \\
\hline Age & 1.054 & $1.027-1.083$ & $<0.001$ \\
Unstable angina & 1.805 & $1.036-3.098$ & 0.034 \\
PTCA history & 2.003 & $1.172-3.384$ & 0.010 \\
LA enlargement & 2.141 & $1.312-3.560$ & 0.003 \\
Dyslipidemia & 0.472 & $0.250-0.844$ & 0.014
\end{tabular}

POAF, postoperative atrial fibrillation; OR, odds ratio; $\mathrm{Cl}$, confidence interval; PTCA, percutaneous transluminal coronary angioplasty; LA, left atrial.

(994 POAF and 5,481 no-POAF) showed that POAF was an independent predictor of long-term mortality (4). In the study by Lee at al. on 1,171 patients who underwent CABG (244 POAF and 927 no POAF), POAF was suggested to be an independent predictor of long-term AF (HR 4.99, 95\% CI: 1.68-14.84, $\mathrm{P}=0.004)$, and long-term survival was shown to be worse in POAF patients $(\mathrm{P}=0.01)$ (5).

However, Levy et al. provided a deep insight regarding research conducted on POAF, suggesting that residual confounders may exist in papers reporting on the hazards of POAF (6). Predictors of POAF such as older age, poor comorbidity and intra-aortic balloon pumps requirement, seem to overlap with those of long-term mortality; i.e., patients who developed POAF tend to be sicker than those without POAF. Therefore, determining the effects of POAF on long-term mortality is inherently challenging with susceptibility to selection bias. Moreover, advanced age has been the only established predictor of POAF; other suggested predictors, including the use of cardiopulmonary bypass in CABG, have not been consistent across papers. Another uncertainty involves the mechanisms in which POAF contributes to long-term adverse outcomes. POAF has been suggested as a risk factor for long-term $\mathrm{AF}$ and long-term mortality $(4,5)$. Although the authors performed adjustments or matching in these studies, POAF patients had higher postoperative complication rates and longer hospital stays. In addition, the onset of AF after surgery, the specific treatment methods of $\mathrm{AF}$, sinus conversion rates and recurrence rates were not described. Recent study by Benedetto et al. also suggested POAF increased risks of late CVA and mortality after isolated CABG. However, the information on the duration of POAF, sinus rhythm conversion rate, recurrence of $\mathrm{AF}$ was not described in the study. In addition, patients with or without POAF showed different preoperative characteristics. Early postoperative complications were not described. Patients in each group had different medications at discharge, especially warfarin. And, non-cardiovascular causes of death were higher than cardiovascular causes (15). Furthermore, it were not mentioned and analyzed in the study, various operative (onpump or off-pump) and graft (use of vein or not, aortano touch or not) strategies were applied in the trial (16). Therefore, it is difficult to analyze the association of POAF with long-term AF and long-term mortality. Whereas, in our study, we tried to inform the onset of POAF and the rate of restoration of sinus rhythm clearly. By applying our protocol-based treatment regimen, the duration of $\mathrm{POAF}$ following OPCAB was a few days to a few hours (mean $1.6 \pm 2.4$ days). Sinus conversion rate was high (97.9\%) and prevalence of persistent AF was low $(n=3)$. Thus, acute POAF might be a potential risk for early stroke and complications. However, the contributing effects of POAF on long-term adverse outcomes remain uncertain and need to be investigated.

Multivariable analysis of our study showed that age, diabetes, unstable angina, PTCA history, and large LA diameter represent the risk factors for POAF. As suggested previously (6), these variables may be consistent with possible risk factors for late adverse events. However, sinus rhythm conversion was achieved in most of our POAF patients, and the follow-up course and nature of the disease may be identical with those without POAF. Therefore, such risk factors may not be contributable for late outcomes. Further investigations for the association of risk factors for POAF-associated long-term outcomes are hence required.

Dyslipidemia revealed as preventive factor for POAF. Several studies suggested that dyslipidemia was inverse associations with AF (17-19). The proposed mechanisms include alterations in cardiac ion channel handling by cholesterol and confounding by hyperthyroidism status and by cardiac load through natriuretic peptides. However, inverse association of dyslipidemia and AF remains uncertain (17). Further studies would be required to evaluate the impacts of dyslipidemia on POAF.

The optimal treatment for POAF has not yet been established. Current guidelines for AF have recommended beta-blockers and calcium channel blockers, but consensus for both therapeutic (11) and prophylactic (12) regimens has not been reached. In addition, guidelines have suggested the consideration of not only sinus rhythm restoration, but also of rate control with anticoagulation therapy (both Class IIa recommendation) (11,12). A recent multi-center randomized 
controlled trial also showed no difference in clinical outcomes between rhythm control and rate control strategies after cardiac surgery (20). However, these suggestions were not specific for POAF after CABG, but for POAF after cardiac surgery which can have different perioperative courses such as the use of cardiopulmonary bypass (CPB) and administration of warfarin after valve operation. Nevertheless, guidelines for $\mathrm{CPB}$ have mentioned the risk and treatment requirements for POAF after $\mathrm{CABG}$, although no specific recommendations have been described $(21,22)$. Our institutional protocol focuses on rhythm control (achieving restoration of sinus rhythm). Patients with documented AF were administrated with amiodarone, and the length of administration was determined by the restoration of sinus rhythm. This protocol worked well in patients with POAF after OPCAB, with up to $98 \%$ rate of sinus rhythm restoration achieved in about 2 days (mean 1.6 \pm 2.4 days). Long-term outcomes did not differ between the POAF and no-POAF groups. Although similar outcomes between rhythm control and rate control of POAF have been suggested (20), guidelines have warned that POAF has associated with a two-fold increase in cardiovascular mortality, future $\mathrm{AF}$ and ischemic stroke compared to patients with sinus rhythm (11). Therefore, we assumed that early sinus rhythm restoration may have contributed to the notable late-outcomes in our study.

Amiodarone is one of the most commonly used medication for AF. However, it may adversely affect various organ systems, including the hepatic, gastrointestinal, pulmonary, thyroid and neurologic systems, the skin and eyes, as well as cause bradycardia (23). Therefore, we proposed the use of lower amiodarone dosages. We used relatively lower doses of amiodarone compared with those used in general practice $(20,24)$, since POAF following OPCAB may be due to increased susceptibility to the triggers of AF after surgery, rather than due to structural substrates present before surgery or surgical trauma (9).

Anticoagulation treatment for POAF is important to prevent thromboembolic events. However, no consensus regimens have been proposed among current guidelines (11-13). The American Association for Thoracic Surgery guidelines have suggested 4-6 weeks of anticoagulation for POAF even after restoration to sinus rhythm (Class I recommendation) (13), to prevent the risk of thromboembolic events due to recurrent AF and LA stunning. Despite these concerns, cardioversion after recent-onset AF (AF duration within 48 hours) did not impair LA velocity (25). Therefore, rapid pharmacological cardioversions may not increase the risk of embolism or recurrent $\mathrm{AF}$. In addition, a proper anticoagulation regimen for POAF after CABG has not been established. At least one antiplatelet therapy is required after CABG. Therefore, the addition of anticoagulants can increase bleeding risks. Investigations on the appropriate regimens for POAF after CABG (such as aspirin and warfarin, or aspirin and NOAC, or dual antiplatelet therapy) are therefore warranted.

Our institutional protocol involves early rhythm control strategies using amiodarone, which achieved successful pharmacological cardioversion in $98 \%$ of POAF patients within a mean of 2 days, and with most patients discharged with sinus rhythm. Additional NOAC or warfarin medications were not required, except for antiplatelet agents for coronary diseases. During follow-up, no significant differences in adverse events were observed between POAF and no-POAF patients.

Our study has several limitations. First, it was a retrospective, non-randomized study from a single institution. Therefore, it may have been influenced by selection bias. We therefore performed IPTW adjustment and propensity score matching analyses to compensate for potential biases in patient selection. However, an unidentified confounding bias may have influenced our results. A prospective, randomized, multicenter study would still be necessary. Second, our study only included patients who underwent OPCAB. Our main institutional operative strategy for CABG is via the off-pump technique to ensure homogeneity in the characteristics of our study cohort. However, our results may not be generalizable to patients who underwent $\mathrm{CABG}$ with $\mathrm{CPB}$ or other cardiac surgeries. Third, we did not perform Holter study during the follow-up period. Therefore, ECGs alone may not capture the full burden of AF.

\section{Conclusions}

Our institutional, amiodarone-based treatment protocol for rapid pharmacological cardioversion of POAF resulted in a high sinus rhythm conversion rate $(97.9 \%)$. The course of $\mathrm{POAF}$ after $\mathrm{OPCAB}$ are usually benign, and the persistence of $\mathrm{AF}$ is rare. Rates of late adverse events, including stroke, were low even without anticoagulation. Regarding the absence of optimal treatment and anticoagulation guidelines for POAF after OPCAB, amiodarone-based treatment protocol may be considered a useful option.

\section{Acknowledgments}

Funding: None. 


\section{Footnote}

Reporting Checklist: The authors have completed the STROBE reporting checklist. Available at https://dx.doi. org/10.21037/jtd-21-466

Data Sharing Statement: Available at https://dx.doi. org/10.21037/jtd-21-466

Conflicts of Interest: All authors have completed the ICMJE uniform disclosure form (available at https://dx.doi. org/10.21037/jtd-21-466). The authors have no conflicts of interest to declare.

Ethical Statement: The authors are accountable for all aspects of the work in ensuring that questions related to the accuracy or integrity of any part of the work are appropriately investigated and resolved. The study was conducted in accordance with the Declaration of Helsinki (as revised in 2013). The study protocol was approved by the hospital's institutional review board, which waived the requirement for patient consent (IRB no: 2020-0643, approval date: 11-18-2020).

Open Access Statement: This is an Open Access article distributed in accordance with the Creative Commons Attribution-NonCommercial-NoDerivs 4.0 International License (CC BY-NC-ND 4.0), which permits the noncommercial replication and distribution of the article with the strict proviso that no changes or edits are made and the original work is properly cited (including links to both the formal publication through the relevant DOI and the license). See: https://creativecommons.org/licenses/by-nc-nd/4.0/.

\section{References}

1. Phan K, Ha HS, Phan S, et al. New-onset atrial fibrillation following coronary bypass surgery predicts long-term mortality: a systematic review and meta-analysis. Eur J Cardiothorac Surg 2015;48:817-24.

2. Suh JH, Park CB, Moon MH, et al. Postoperative Atrial Fibrillation after Isolated Coronary Artery Bypass Graft Surgery. Korean J Thorac Cardiovasc Surg 2009;42:14-21.

3. Yoo KJ, Ko YH, Lim SH, et al. Analysis of risk factors of atrial fibrillation after coronary artery bypass grafting. Korean J Thorac Cardiovasc Surg 1996;29:599-605.

4. Villareal RP, Hariharan R, Liu BC, et al. Postoperative atrial fibrillation and mortality after coronary artery bypass surgery. J Am Coll Cardiol 2004;43:742-8.

5. Lee SH, Kang DR, Uhm JS, et al. New-onset atrial fibrillation predicts long-term newly developed atrial fibrillation after coronary artery bypass graft. Am Heart J 2014;167:593-600.e1.

6. Levy D, Kannel WB. Postoperative atrial fibrillation and mortality: do the risks merit changes in clinical practice? J Am Coll Cardiol 2004;43:749-51.

7. Greenberg JW, Lancaster TS, Schuessler RB, et al. Postoperative atrial fibrillation following cardiac surgery: a persistent complication. Eur J Cardiothorac Surg 2017;52:665-72.

8. Lomivorotov VV, Efremov SM, Pokushalov EA, et al. New-Onset Atrial Fibrillation After Cardiac Surgery: Pathophysiology, Prophylaxis, and Treatment. J Cardiothorac Vasc Anesth 2016;30:200-16.

9. Echahidi N, Pibarot P, O'Hara G, et al. Mechanisms, prevention, and treatment of atrial fibrillation after cardiac surgery. J Am Coll Cardiol 2008;51:793-801.

10. Obadia JF, el Farra M, Bastien OH, et al. Outcome of atrial fibrillation after mitral valve repair. J Thorac Cardiovasc Surg 1997;114:179-85.

11. Kirchhof P, Benussi S, Kotecha D, et al. 2016 ESC Guidelines for the management of atrial fibrillation developed in collaboration with EACTS. Eur Heart J 2016;37:2893-962.

12. January CT, Wann LS, Alpert JS, et al. 2014 AHA/ ACC/HRS guideline for the management of patients with atrial fibrillation: a report of the American College of Cardiology/American Heart Association Task Force on practice guidelines and the Heart Rhythm Society. Circulation 2014;130:e199-267.

13. Frendl G, Sodickson AC, Chung MK, et al. 2014 AATS guidelines for the prevention and management of perioperative atrial fibrillation and flutter for thoracic surgical procedures. J Thorac Cardiovasc Surg 2014;148:e153-93.

14. Austin PC. The use of propensity score methods with survival or time-to-event outcomes: reporting measures of effect similar to those used in randomized experiments. Stat Med 2014;33:1242-58.

15. Benedetto U, Gaudino MF, Dimagli A, et al. Postoperative Atrial Fibrillation and Long-Term Risk of Stroke After Isolated Coronary Artery Bypass Graft Surgery. Circulation 2020;142:1320-9.

16. Taggart DP, Lees B, Gray A, et al. Protocol for the Arterial Revascularisation Trial (ART). A randomised trial to compare survival following bilateral versus single 
internal mammary grafting in coronary revascularisation [ISRCTN46552265]. Trials 2006;7:7.

17. Guan B, Li X, Xue W, et al. Blood lipid profiles and risk of atrial fibrillation: A systematic review and meta-analysis of cohort studies. J Clin Lipidol 2020;14:133-42.e3.

18. Mora S, Akinkuolie AO, Sandhu RK, et al. Paradoxical association of lipoprotein measures with incident atrial fibrillation. Circ Arrhythm Electrophysiol 2014;7:612-9.

19. Lopez FL, Agarwal SK, Maclehose RF, et al. Blood lipid levels, lipid-lowering medications, and the incidence of atrial fibrillation: the atherosclerosis risk in communities study. Circ Arrhythm Electrophysiol 2012;5:155-62.

20. Gillinov AM, Bagiella E, Moskowitz AJ, et al. Rate Control versus Rhythm Control for Atrial Fibrillation after Cardiac Surgery. N Engl J Med 2016;374:1911-21.

21. Neumann FJ, Sousa-Uva M, Ahlsson A, et al. 2018 ESC/ EACTS Guidelines on myocardial revascularization. Kardiol Pol 2018;76:1585-664.

Cite this article as: Lee H, Kim HJ, Yoo JS, Kim DJ, Yeom SY, Cho KR. Early pharmacologic conversion of atrial fibrillation after off-pump coronary artery bypass grafting. J Thorac Dis 2021;13(7):4072-4082. doi: 10.21037/jtd-21-466
22. Hillis LD, Smith PK, Anderson JL, et al. 2011 ACCF/ AHA Guideline for Coronary Artery Bypass Graft Surgery. A report of the American College of Cardiology Foundation/American Heart Association Task Force on Practice Guidelines. Developed in collaboration with the American Association for Thoracic Surgery, Society of Cardiovascular Anesthesiologists, and Society of Thoracic Surgeons. J Am Coll Cardiol 2011;58:e123-210.

23. Vorperian VR, Havighurst TC, Miller S, et al. Adverse effects of low dose amiodarone: a meta-analysis. J Am Coll Cardiol 1997;30:791-8.

24. Raiten J, Patel PA, Gutsche J. Management of postoperative atrial fibrillation in cardiac surgery patients. Semin Cardiothorac Vasc Anesth 2015;19:122-9.

25. Antonielli E, Pizzuti A, Dogliani S, et al. Absence of left atrial stunning after cardioversion of recent-onset atrial fibrillation in patients at low-stroke risk. Eur J Emerg Med 2017;24:217-23. 
Supplementary

Table S1 Baseline characteristics after propensity score-matching

\begin{tabular}{|c|c|c|c|c|}
\hline Variables & No-POAF (n=91) & POAF $(n=91)$ & $P$ value & SMD \\
\hline Age, years & $68.0(60.5-73.0)^{b}$ & $68.0(62.0-73.5)^{\mathrm{b}}$ & & \\
\hline Sex, female, n (\%) & $26(28.6)$ & $24(26.4)$ & 0.874 & 0.049 \\
\hline $\mathrm{BSA}, \mathrm{m}^{2}$ & $1.71 \pm 0.16^{\mathrm{a}}$ & $1.71 \pm 0.18^{\mathrm{a}}$ & 0.897 & 0.018 \\
\hline \multirow[t]{2}{*}{ BMI, $\mathrm{kg} / \mathrm{m}^{2}$} & $24.6 \pm 2.3^{\mathrm{a}}$ & $24.7 \pm 2.7^{\mathrm{a}}$ & 0.788 & 0.038 \\
\hline & $24.7(23.0-25.9)^{\mathrm{b}}$ & $24.3(23.0-26.6)^{b}$ & & \\
\hline Hypertension, n (\%) & $60(65.9)$ & $63(69.2)$ & 0.742 & 0.070 \\
\hline Diabetes mellitus, n (\%) & $52(57.1)$ & $55(60.4)$ & 0.749 & 0.067 \\
\hline NYHA class III-IV, n (\%) & $4(4.4)$ & $4(4.4)$ & $>0.999$ & $<0.001$ \\
\hline CCS class 4, n (\%) & $8(8.8)$ & $6(6.6)$ & 0.789 & 0.083 \\
\hline Preoperative beta-blocker use, n (\%) & $66(72.5)$ & $68(74.7)$ & 0.871 & 0.050 \\
\hline Hyperthyroidism, n (\%) & $2(2.2)$ & $0(0.0)$ & 0.477 & 0.212 \\
\hline Chronic renal failure, n (\%) & $4(4.4)$ & $6(6.6)$ & 0.752 & 0.097 \\
\hline COPD, n (\%) & $3(3.3)$ & $4(4.4)$ & $>0.999$ & 0.057 \\
\hline Interstitial pneumonia, n (\%) & $2(2.2)$ & $0(0.0)$ & 0.477 & 0.212 \\
\hline $\mathrm{CHA}_{2} \mathrm{DS}_{2}-\mathrm{VAS}_{\mathrm{C}}$ score & $2.9 \pm 1.5^{\mathrm{a}}$ & $3.0 \pm 1.4^{\mathrm{a}}$ & 0.702 & 0.053 \\
\hline PTCA history, n (\%) & $25(27.5)$ & $29(31.9)$ & 0.627 & 0.096 \\
\hline $\mathrm{EF}<40, \mathrm{n}(\%)$ & $17(18.7)$ & $16(17.6)$ & $>0.999$ & 0.029 \\
\hline LA enlargement, n (\%) & $61(67.0)$ & $62(68.1)$ & $>0.999$ & 0.023 \\
\hline
\end{tabular}

${ }^{\mathrm{a}}$ Mean \pm standard deviation; ${ }^{\mathrm{b}}$ Median (Q1-Q3). POAF, postoperative atrial fibrillation; SMD, standardized mean difference; BSA, body surface area; BMI, body mass index; NYHA, New York Heart Association; CCS, Canadian Cardiovascular Society; COPD, chronic obstructive pulmonary disease; POAD, peripheral occlusive arterial disease; OMI, old myocardial infarction; PTCA, percutaneous transluminal coronary angioplasty; EF, ejection fraction; LA, left atrial. 
Table S2 Operative data after propensity score-matching

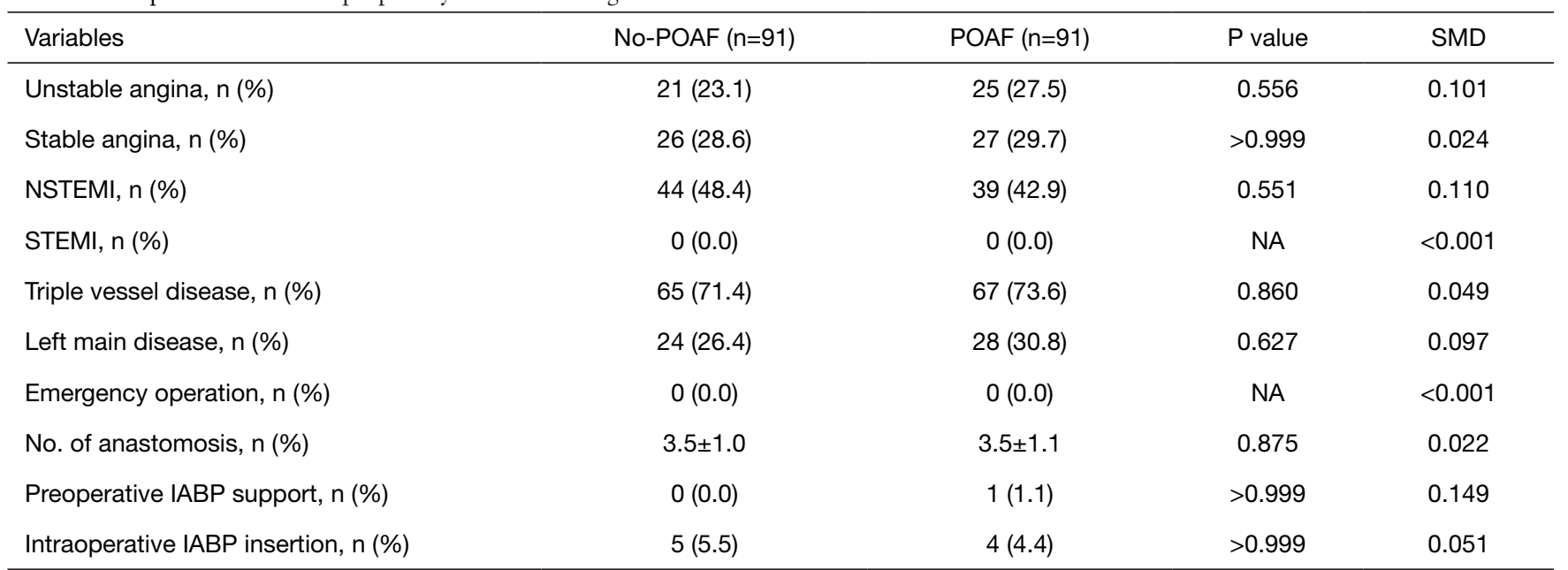

POAF, postoperative atrial fibrillation; SMD, standardized mean difference; NSTEMI, non-ST-elevation myocardial infarction; STEMI, ST-elevation myocardial infarction; IABP, intra-aortic balloon pump. 